\title{
Metamaterial Composite with an Ultra-Broadband Usable Range of over 25 Terahertz
}

Strikwerda, Andrew; Zalkovskij, Maksim; Krabbe, Alexander; Lorenzen, Dennis Lund; Lavrinenko, Andrei; Jepsen, Peter Uhd

Published in:

Proceedings of 2014 Conference on Lasers and Electro-Optics (CLEO)

Publication date:

2014

Document Version

Publisher's PDF, also known as Version of record

Link back to DTU Orbit

Citation $(A P A)$ :

Strikwerda, A., Zalkovskij, M., Krabbe, A., Lorenzen, D. L., Lavrinenko, A., \& Jepsen, P. U. (2014). Metamaterial Composite with an Ultra-Broadband Usable Range of over 25 Terahertz. In Proceedings of 2014 Conference on Lasers and Electro-Optics (CLEO) [STU2F.1] IEEE.

\section{General rights}

Copyright and moral rights for the publications made accessible in the public portal are retained by the authors and/or other copyright owners and it is a condition of accessing publications that users recognise and abide by the legal requirements associated with these rights.

- Users may download and print one copy of any publication from the public portal for the purpose of private study or research.

- You may not further distribute the material or use it for any profit-making activity or commercial gain

- You may freely distribute the URL identifying the publication in the public portal 


\title{
Metamaterial Composite with an Ultra-Broadband Usable Range of over 25 Terahertz
}

\author{
Andrew C. Strikwerda", Maksim Zalkovskij, Alexander Krabbe, Dennis Lund Lorenzen, Andrei V. \\ Lavrinenko, and Peter Uhd Jepsen \\ DTU Fotonik - Department of Photonics Engineering, Technical University of Denmark, DK-2800 Kgs. Lyngby, Denmark \\ "astr@fotonik.dtu.dk
}

\begin{abstract}
Using a metamaterial composite, we demonstrate a bandpass filter that has only a single transmission mode from 0 to $>25 \mathrm{THz}$. This usable bandwidth matches, or exceeds, that of currently used THz sources.

OCIS codes: (160.3918) Metamaterials; (230.7408) Wavelength filtering devices; (300.6495) Spectroscopy, terahertz.
\end{abstract}

\section{Introduction}

Since their introduction nearly fifteen years ago, metamaterials have provided a unique design paradigm [1]. However, their use is often limited to a small bandwidth around their design frequency due to the resonant nature of most metamaterials, appearance of unwanted higher order modes, and prevalence of the effective medium approximation where metamaterials are only considered to have a "designed" permittivity and permeability in the deep sub-wavelength regime. While this narrowband behavior can be easily integrated with continuous wave sources and applications, the two most common methods of THz generation, photoconductive antennas and nonlinear generation in either crystals or air plasma, both yield broad spectra that suggest there is an unfulfilled need for broadband $\mathrm{THz}$ components.

Here we reconcile the disconnect between traditional broadband sources and narrowband metamaterial components by demonstrating a metamaterial composite with an ultra-broadband usable range. As a proof of principle of this ultra-broadband concept, we have made a series of band pass filters that, to the best of our knowledge, display the largest usable bandwidth of any metamaterial device to date. The filters have a single pass band, with a central frequency scalable from $0.86-8.51 \mathrm{THz}$, while rejecting all other frequency components up to $>25 \mathrm{THz}$.
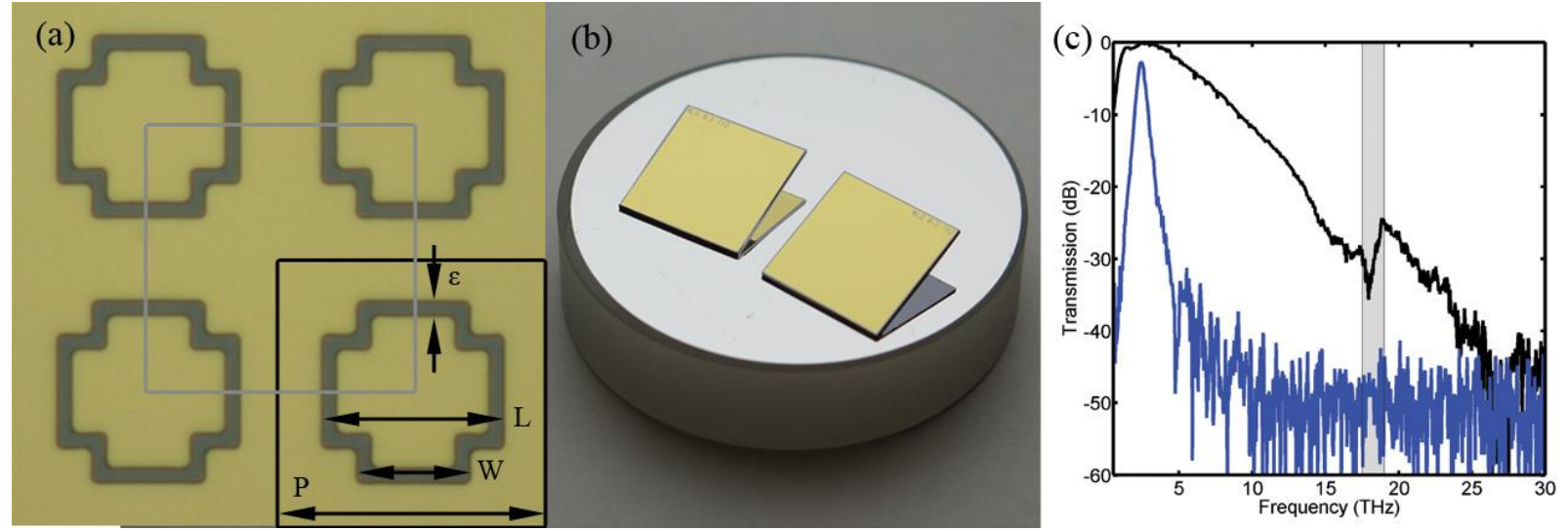

Figure 1 - Optical pictures of fabricated samples. (a) a 2x2 unit picture of the sample. (b) A single-sided sample (right) and a double-sided sample (left). The dimensions are $\mathrm{L}=7.09^{*} \sigma ; \mathrm{W}=4.5^{*} \sigma ; \mathrm{P}=10.03^{*} \sigma$; and $\varepsilon=1.5$ where all dimensions are in microns. (c) Experimental transmission of a $\sigma=2.5$ filter (blue) and a reference pulse through air (black). The grey area is a silicon phonon resonance that is intrinsic to the system.

\section{Metamaterial Composite}

The filter itself is a metamaterial composite of two distinct components. The first is a metamaterial cross structure, and the second, depending upon your perspective, is either a Babinet complement [2] or a metal mesh [3]. To clearly identify these constituents, we present an optical picture of a 2x2 array of unit cells in Figure 1(a). The unit cell in the bottom right, outlined in black, clearly identifies the metamaterial cross component at the center of the unit cell. However, this unit cell choice suggests that the cross is placed inside of its own, slightly larger, Babinet complement. If the unit cell is translated by $(-1 / 2,1 / 2) \times \mathrm{P}$, to the grey outlined unit cell, a different structure is suggested. In this new unit cell, if the cross is ignored, it can be seen that the Babinet complement is also a metal mesh filter. Metal meshes, which have been widely used since their introduction by Ulrich [3], can be described as 
inductive and capacitive meshes. A similar structure was presented previously [4], although we have expanded upon the bandwidth by an order of magnitude.

The sample dimensions - except for $\varepsilon$, which was held constant - were all scaled by a dimensionless parameter, $\sigma$, and can be found in the caption for Figure 1. Ten different samples with varying values of $\sigma$ were fabricated, all on high resistivity $\mathrm{Si}$, spanning the range of $0.86-8.51 \mathrm{THz}$. Furthermore, the samples were fabricated in singleand double-sided configurations, as shown in Fig 1(b).

\section{Results and discussion}

The samples were measured with THz-time domain spectroscopy using a two-color air plasma for generation and air biased coherent detection (ABCD), resulting in an ultra-broadband frequency coverage, spanning $0.1-30$ THz. The computer simulations were conducted using CST Microwave Studios.

An aggregate comparison between simulation and experiment is shown in Figure 2. The two plots compare the central frequency $\left(f_{0}\right)$ and full width at half maximum (FWHM) versus $\sigma$ for both the single- and double-sided samples. Figure 2(a) also has a line that is simply the $\sigma=1$ central frequency scaled linearly with $\sigma$. If $\varepsilon$ was scaled with $\sigma$, instead of kept constant, every dimension would be scaled identically and this line would predict $f_{0}$ for all $\sigma$ due to the scale invariance of Maxwell's equations. However, this would either reduce the filter performance or the fabrication would be practically unfeasible. Fits to the data reveal the actual scaling versus $\sigma: f_{0}=8.223 \sigma^{-1.424}+$ $0.2774 ; \Delta f_{1}=5.511 \sigma^{-1.557}+0.2178 ; \Delta f_{2}=3.161 \sigma^{-1.046}-0.2098 . \Delta f_{1}$ and $\Delta f_{2}$ are the FWHM for the single- and double-sided samples, respectively.

The effect of the single- versus double-sided structures can clearly be seen in Figure 2(b). The peak transmission through the bandpass structures is roughly equivalent to the Fresnel transmission coefficient for the air HR-Si interface. Subsequently, the additional layer on the backside of the Si wafer further reduces the transmitted bandwidth without significantly affecting its peak performance.

(a) $f_{0}$

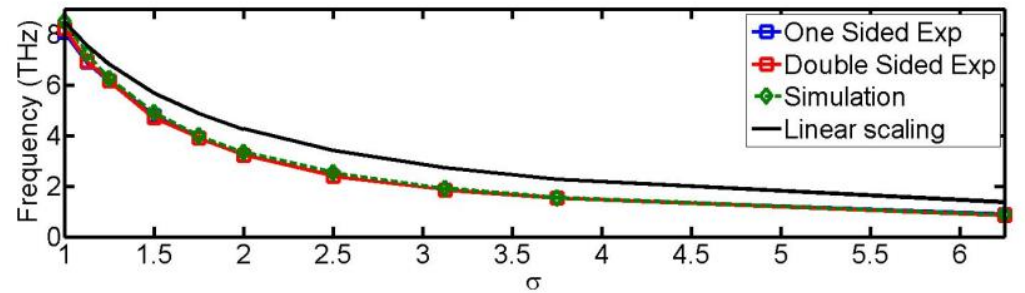

(b) $\Delta f$

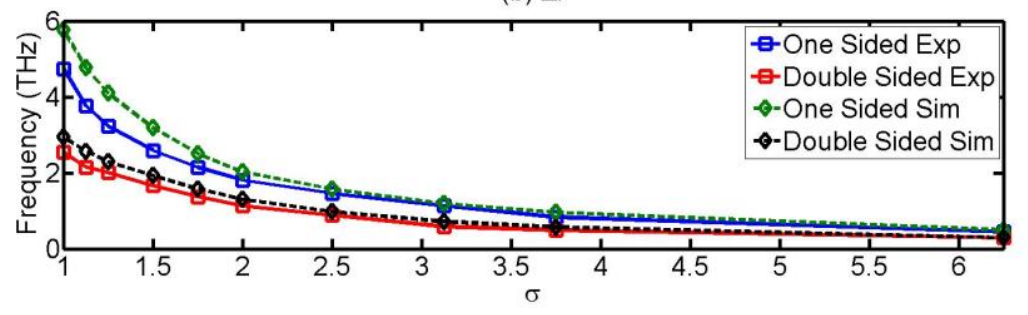

Figure 2: (a) The aggregate agreement between $f_{0}$ in simulation and experiment. To highlight the effect of the constant minimum linewidth in all structures, the solid black line represents a linear scaling of $f_{0}$ vs $\sigma$. (b) The comparison of the FWHM for experimental and simulated results.

\section{Conclusion}

In conclusion, we have presented a metamaterial composite with an ultra-broadband usable bandwidth suitable for virtually any $\mathrm{THz}$ source. We have constructed a series of band pass filters that clearly demonstrate this concept, and provided simple equations that can be used to construct frequency filters from $0.86-8.51 \mathrm{THz}$ without need for simulation or design. It is our hope that this work will bring an easy to fabricate, functional $\mathrm{THz}$ component to the laboratory, and expand the reach of metamaterial based $\mathrm{THz}$ components towards broadband functional components.

\section{References}

[1] W. Withayachumnankul and D. Abbott, "Metamaterials in the Terahertz Regime," Photonics J., IEEE 1, 99-118 (2009).

[2] H-T Chen, J. O'Hara, A.J. Taylor, R.D. Averitt, C. Highstrete, M. Lee, and W.J Padilla, "Complementary planar terahertz metamaterials,"

Opt. Ex. 15, 1084-1095 (2007).

[3] R. Ulrich, "Far-infrared properties of metallic mesh and its complementary structure," Infrared Phys 7, $37-55$ (1967).

[4] O. Paul, R. Beigang, and M. Rahm, "Highly selective terahertz bandpass filters based on trapped mode excitation," Opt. Ex. 17, 18590-5

(2009). 